\title{
Evidence for a supercooled plastic-crystal phase in solid ethanol
}

\author{
A. Srinivasan and F. J. Bermejo \\ Instituto de Estructura de la Materia, Consejo Superior de Investigaciones Científicas, Serrano 123, E-28006 Madrid, Spain \\ A. de Andrés \\ Instituto de Ciencia de Materiales de Madrid, Sede B, Consejo Superior de Investigaciones Científicas, \\ Universidad Autónoma de Madrid, Facultad de Ciencias, C-IV, Cantoblanco, E-28049 Madrid, Spain \\ J. Dawidowski \\ Instituto de Estructura de la Materia, Consejo Superior de Investigaciones Científicas, Serrano 123, E-28006 Madrid, Spain \\ J. Zúñiga \\ Departamento de Física de la Materia Condensada, Universidad del Pais Vasco, Euskal Herriko Universitatea, \\ Facultad de Ciencias, E-48960 Bilbao, Spain \\ A. Criado \\ Departamento de Física de la Materia Condensada, Universidad de Sevilla, and Instituto de Ciencia de Materiales, \\ Consejo Superior de Investigaciones Científicas, Facultad de Ciencias, P.O. Box 1065, E-41080 Sevilla, Spain
}

(Received 18 October 1995; revised manuscript received 8 December 1995)

\begin{abstract}
The existence of an orientationally disordered cubic phase of solid ethanol is revealed by x-ray diffraction and Raman spectroscopic measurements. Such a phase, whose existence was postulated some time ago on the basis of specific-heat measurements, is produced by quenching below some $95 \mathrm{~K}$ a plastic crystal formed upon melting and subsequent annealing of the topologically disordered (glassy) solid. The relevance of the present findings for current discussions on glassy dynamics is analyzed in some detail.
\end{abstract}

Despite recent advances in our understanding of the microscopic mechanisms driving the dynamics of glasses at intermediate (above $1 \mathrm{~K}$ ) temperatures, ${ }^{1}$ most studies regarding topologically disordered solids (i.e., real glassy materials) still encounter substantial difficulties when trying to go beyond descriptions of the data by means of phenomenological approaches. This contrasts with a more developed understanding regarding the dynamics of disordered crystals formed by either mixed ${ }^{2}$ or chemically homogeneous (plastic and glassy crystals ${ }^{3,4}$ ) materials, which in common with structural glasses exhibit some features considered as fingerprints of the glassy dynamics. Such developments are, however, restricted to materials which being disordered in some quantity (chemical composition, relative molecular orientations, etc.) still have an underlying crystal lattice, thus enabling the calculation of a number of physical properties (i.e., coupling between molecular translations and rotations, softening of elastic constants due to disorder, anomalous dielectric behavior, etc.) in terms of well-established techniques. ${ }^{2}$ The relevance of such findings for the understanding of real glasses was not explored in detail, perhaps due to the fact that most of the existing materials can either be converted into topological or disordered-crystal states, exceptions to such a rule being rare (amorphous and hexagonal ice being an example which involves rather different routes of preparation which pose severe conditions when trying to compare the two in terms of transition temperatures).

The existence of some polymorphic phase in solid ethanol which could be prepared under mild thermal conditions was known to exist since the work of Haida et al. ${ }^{5}$ who observed calorimetric anomalies distinct from those associated with the glass transition $\left(T_{g}=97 \mathrm{~K}\right)$, the melting of the stable crystal $\left(T_{m}=169 \mathrm{~K}\right)$, or crystallization of the supercooled liquid into such a phase $\left(T_{\mathrm{cr}} \approx 125 \mathrm{~K}\right)$. In fact, the presence of a well-defined exothermic anomaly at some $115 \mathrm{~K}$ was interpreted as the onset of a crystal phase (crystal II) which once grown exhibited a calorimetric transition into another crystalline phase (glassy crystal), showing small differences in heat capacity with those of the glass obtained by rapid cooling of the normal liquid, but evidencing a calorimetric entropy between that of the glass and the stable crystal. A NMR relaxation measurement reported later on ${ }^{6}$ showed that the dynamics of the real glass and that of the glassy crystal as sampled by the values of the second moment of the NMR shared some analogies, whereas inspection of the relative values of the relaxation times revealed some systematic differences between both.

Our aim here is to establish the nature of the crystal II and glassy crystal phases of this material. If, as expected, a supercooled plastic crystal (SPC) can be formed by freezing the rotational freedom of the plastic crystal (PC), it would then provide an excellent benchmark material where quantitative studies on the role of different degrees of freedom (positional and orientational) on the dynamics of the glass at intermediate temperatures could be carried in detail.

Spectroscopic grade ethanol was used without further purification, avoiding exposure to atmosphere to prevent capture of water vapour. The x-ray diffraction measurements were performed using a Stoe diffractometer, using the $\mathrm{Cu}$ $K \alpha$ line $\lambda=1.5418 \AA$, being the samples contained in glass 


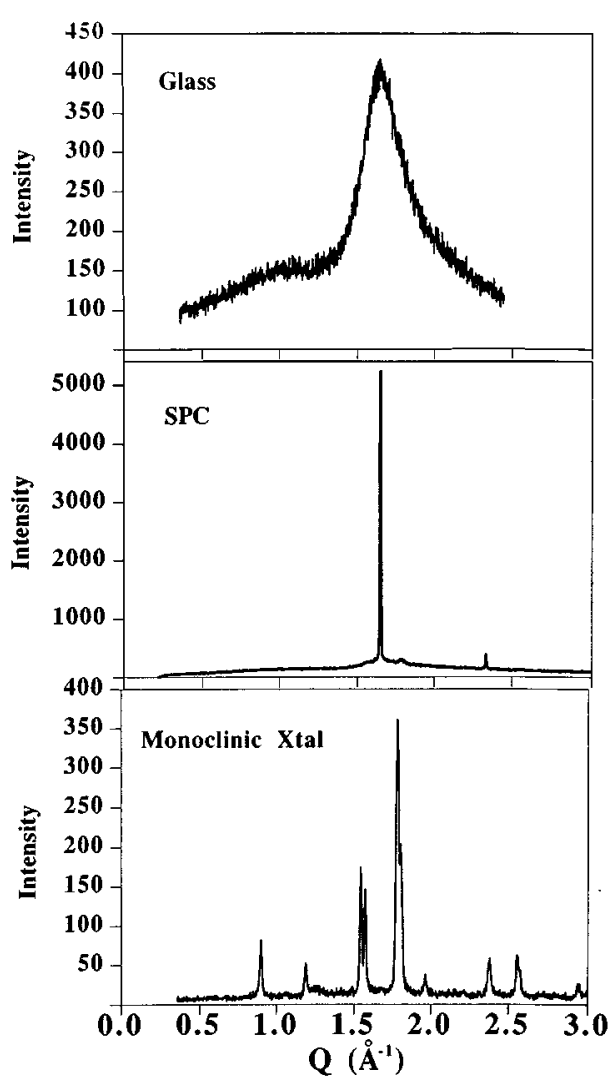

FIG. 1. X-ray diffraction patterns of (a) the positionally disordered glass at $80 \mathrm{~K}$, (b) the supercooled cubic crystal phase at $80 \mathrm{~K}$, and (c) the stable (monoclinic) crystal at $120 \mathrm{~K}$. The small peak at $Q \approx 1.786 \AA^{-1}$ in (b) comes from a small contamination of the monoclinic crystal phase.

capillaries cooled by an Oxford Instruments cryostream cooler. The Raman spectra were recorded on an $X-Y$ Dilor spectrometer equipped with an enhanced photodiode array. Slit widths giving nominal resolutions of 1.8 and $3.7 \mathrm{~cm}^{-1}$ were employed for crystal and glass spectra, respectively. Excitation was achieved by an $\mathrm{Ar}^{+}$source lasing at $514 \mathrm{~nm}$ and delivering some $10 \mathrm{~mW}$ to the sample which was contained within $5 \mathrm{~mm}$ quartz tubes. These were kept within a Oxford Instruments flux cryostat used to keep temperatures between $10 \mathrm{~K}$ and ambient. The scattered light was collected on the Stokes side at right angles.

Sample preparation regarding the normal glass and stable (monoclinic) crystal phases is straightforward since it only involves a quench below $T_{g}=97 \mathrm{~K}$ at a rate not slower than $6 \mathrm{~K} \mathrm{~min}^{-1}$ (glass) and a subsequent annealing at some 130 $\mathrm{K}$ for at least a half hour (crystal). As regards the preparation of the supercooled-plastic-crystal phase, our own experience indicates that reproducible results are obtained if the supercooled liquid formed upon melting the normal glass is allowed to anneal for some minutes at $T=100 \mathrm{~K}$ followed by a rapid quench below the $T_{a}=97 \mathrm{~K}$ transition. A macroscopic phase separation occurs during annealing where a translucent solid phase segregates from the clear liquid.

Figure 1 shows a plot comparing the x-ray scattered intensities for the three phases of solid ethanol. The monoclinic crystal spectrum is fully compatible with the crystal structure described in Ref. 7 which corresponds to a unit cell contain- ing four molecules, two of which are independent in the asymmetric unit of $P c$ symmetry. The referred two molecules have a different internal structure (conformation), showing dihedral $\mathrm{C}-\mathrm{C}-\mathrm{O}-\mathrm{H}$ angles of some $297^{\circ}$ (gauche) and $179^{\circ}$ (trans) with also significant variation of the bond-angle values. ${ }^{7}$ The crystal structure shows infinitely bonded zigzag chains joined by $\mathrm{O}-\mathrm{H} \cdots \mathrm{O}$ bonds of length $2.716 \AA$. In full contrast, the glass shows a broad spectrum with a main diffraction peak centered at $Q \approx 1.65 \AA^{-1}$ and a width of $\approx 0.5 \AA^{-1}$. The parameters of such a peak are close to those found for a chemically related glass (methanol) discussed in some detail in the past, ${ }^{8}$ and which were found to represent ${ }^{9}$ a system of long, winding chains, with a correlation length [defined by the extent in real space where the $g(r)$ intermolecular distribution function shows noticeable oscillations] of some $15 \AA$. The existence of a high-symmetry crystal phase is demonstrated by the diffractogram of Fig. 1 (middle), which shows that most of the intensity arises from two Bragg reflections at $Q=1.651 \AA^{-1}$ (intense) and $Q=2.335 \AA^{-1}$ (weak), alongside with small traces of contamination by minute amounts of the monoclinic phase and a broad, diffuse background. Such a diffraction pattern can be understood as arising from a body-centered-cubic structure with lattice constant $a=5.381 \AA$, the (110) and (200), respectively, being the observed reflections. Assuming two molecules per unit cell, the assignment would correspond to a solid with a macroscopic density of $982.5 \mathrm{~kg} \mathrm{~m}^{-3}$, close to the value at $T_{g}$ as extrapolated from tabulated values for the normal supercooled liquids ${ }^{10}\left(\rho=958.2 \mathrm{~kg} \mathrm{~m}^{-3}\right)$. The value for the lattice constant mentioned above becomes very close to that of 5.377(4) $\AA$ corresponding to the $a$ dimension of the monoclinic cell. ${ }^{7}$ On the other hand, the close location of the (110) reflection with the wave vector characteristic of the peak maximum in the glass phase provides a clear example of the physical meaning of the first diffraction peak in glasses, an issue which was the focus of some recent controversies. ${ }^{11} \mathrm{In}$ fact, comparison of diffractograms of glass and cubic crystal clearly suggests that only the width of such a peak should bear a clear physical significance in a glass.

Because of the small number of observed Bragg reflections, further details regarding the structure of such a cubic phase could only be derived from additional independent measurements. Between those, a comparison of the Raman spectra of the solid within its three (cubic, monoclinic, and glass) phases was chosen and the most relevant results are displayed in Fig. 2. The immediate consequence of such a comparison regards the existence of a strong static disorder in the cubic phase as demonstrated by the absence of wellresolved phonon peaks below some $40 \mathrm{meV}$. That such a disorder is of static (orientational) nature is further ratified by the absence of any thermal anomaly for this phase below our actual $T=80 \mathrm{~K}$, which should be present if substantial rotational motions were still present. It seems then clear that the cubic phase represents a SPC or glassy crystal phase, where the large rotational motions present above $97 \mathrm{~K}$ have frozen into a strongly orientationally disordered phase. In fact, spectra measured during annealing time show in addition to the peaks shown in the SPC phase an additional strong diffuse background. The amount and kind of disorder (i.e., statistical or through some preferential direction) cannot be inferred from the available data, although the similitude between the 


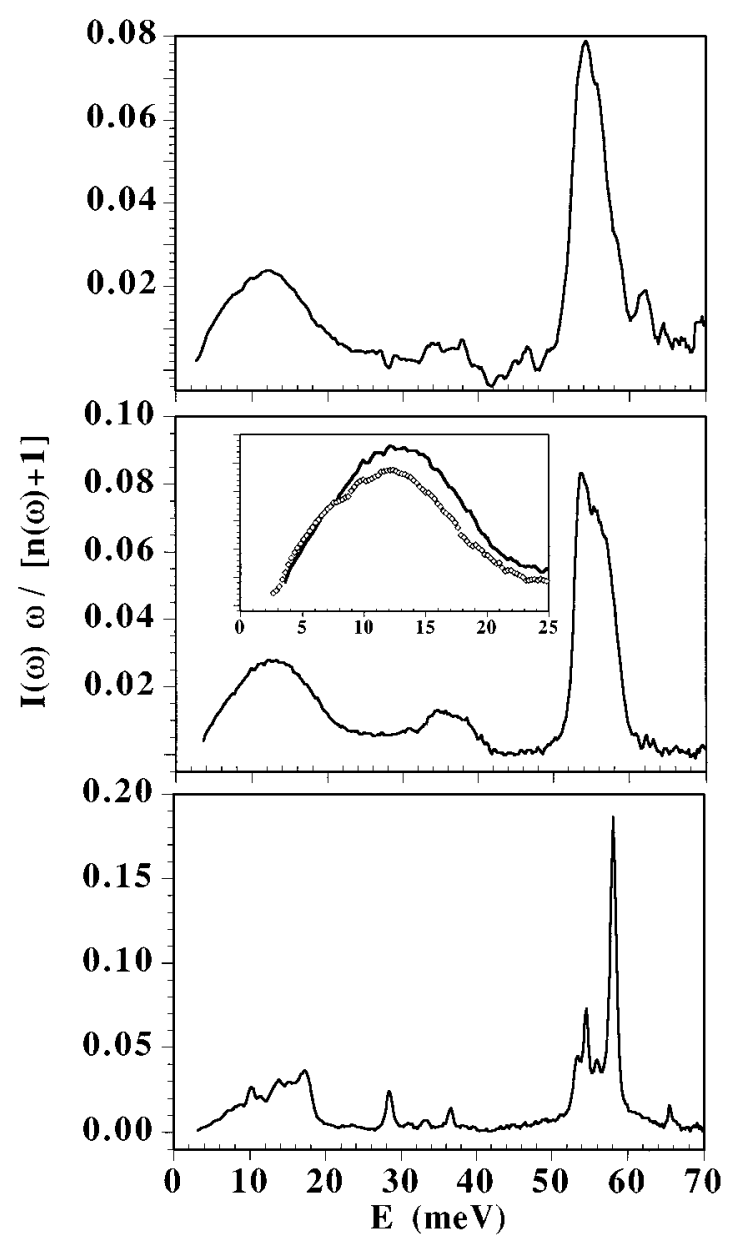

FIG. 2. Raman frequency distributions $Z_{R}(\omega)$ $=I(\omega) \omega /[n(\omega)+1]$ at $10 \mathrm{~K}$ for (a) the glass phase, upper frame (b) the SPC phase midframe, and (c) the monoclinic crystal. The inset in (b) represents an enlargement of the frequency distributions of the glass and SPC phases. Intensities are given in arbitrary units.

low-frequency Raman spectra (below some $20 \mathrm{meV}$ ) and the coincidence of the position of the intense Bragg reflection with the maximum of $S(Q)$ in the canonical glass pinpoints the existence of remarkable correlations between molecular orientations as it is known to occur in the glass phase of other alcohols. ${ }^{9,12}$

The Raman spectra of the glass and SPC solid show rather similar shapes of the frequency distributions. Because of the isotropy of the glass and the orientational disorder in the SPC, the Raman intensities could be considered to be directly related to the vibrational density of states through the usual expression $Z_{R}(\omega) \propto I(\omega) \omega /[n(\omega)+1]$, where in the absence of additional information a quadratic frequency dependence for the $C(\omega)$ light-to-vibration coupling function is assumed (i.e., complete randomness of couplings). Notice from the inset in Fig. 2 that the main difference between canonical and SPC spectra regards the position of the maxima which in the SPC phase is shifted some $1 \mathrm{meV}$ above that of the canonical glass, as well as a rather small excess of modes below $6 \mathrm{meV}$ of the glass with respect to the SPC.

An assessment of the physical soundness of such results is provided by evaluating the harmonic contribution to the constant-volume heat capacity from the $Z_{R}(\omega)$ estimate of the vibrational densities of states described above. To avoid problems regarding the normalization of $Z_{R}(\omega)$ to absolute units only the ratios $C_{v}^{g} / C_{v}^{\mathrm{SPC}}$ of heat capacities for the glass and SPC are considered. Such a quantity yields a value of 1.34 to be compared with that of 1.60 derived from data given in Ref. 13. On the other hand, the maxima of $C_{v}(T) / T^{3}$ curves appear in the SPC phase shifted some 1.8 $\mathrm{K}$ above that for the structural glass, a fact also borne out by calorimetric measurements where the maxima of the SPC sample is located at some $6.5 \mathrm{~K}$ whereas that for the glass appears at $\approx 4.5 \mathrm{~K}$.

In consequence, and with the above provisos in mind, it seems clear that the cubic SPC phase of ethanol here studied corresponds to the same one explored by calorimetric means. ${ }^{5,13}$ This being the case, the difference in specific heats at $T_{g}$ discussed by Johari and others ${ }^{14}$ upon melting the glass and that arising from the SPC-plastic crystal transition can be rationalized on a more quantitative basis. In fact, comparison of the jumps in $C_{p}$ at the glass transition and rotational arrest points $T_{g}=T_{a}=97 \mathrm{~K}$ of 35 and $23 \mathrm{~J} \mathrm{~K}^{-1} \mathrm{~mol}^{-1}$ evidences that some $66 \%$ of the jump upon melting the glass can be accounted by the onset of rapid molecular rotations. Such a percentage becomes even larger (75\%) for the jump in entropy, and goes to some $63 \%$ for the configurational entropy.

In conclusion, the existence of a orientationally disordered phase in a system considered since many decades ago as one of the clearest examples of a glass-forming liquid has been demonstrated. From the present data as well as from that already available, the SPC solid can be rightly considered as a phase with an intermediate kind of disorder between the ground state (monoclinic crystal) and the glass. In this respect, its detailed study will open up the possibility of separating the effects of positional and orientational disorder on different magnitudes usually considered in studies on glassy dynamics.

This work was supported in part by DGICYT Grant No. PB92-0114-C04.
${ }^{1}$ For a recent compendium of discussions on this topic, see Proceedings of the II International Discussion Meeting on Relaxation in Complex Systems, edited by K.L. Ngai et al. [J. NonCryst. Solids 172-174, (1994)].

${ }^{2}$ R.M. Lynden-Bell and K.H. Michel, Rev. Mod. Phys. 66, 721 (1994).
${ }^{3}$ The Plastic Crystalline State, edited by J.N. Sherwood (Wiley, New York, 1979).

${ }^{4}$ A. Loidl and R. Böhmer, in Disorder Effects on Relaxational Processes, edited by R. Richert and A. Blumen (Springer-Verlag, Berlin, 1994), p. 659.

${ }^{5}$ O. Haida, H. Suga, and S. Seki, J. Chem. Thermodyn. 9, 1133 
(1977). Also M. Hugo, K. Schlüter, and A. Würflinger, Z. Phys. Chem. (Neue Folge) 175, 235 (1992).

${ }^{6}$ T. Eguchi, G. Soda, and H. Chihara, Mol. Phys. 40, 681 (1980).

${ }^{7}$ P.G. Jönsson, Acta Crystallogr. B 32, 232 (1976).

${ }^{8}$ F.J. Bermejo, A. Criado, M. Garcia-Hernandez, J. Alonso, C. Prieto, and J.L. Martinez, J. Phys. Condens. Matter 6, 405 (1994), and references therein.

${ }^{9}$ F.J. Bermejo, J. Alonso, A. Criado, F.J. Mompean, J.L. Martinez, M. Garcia-Hernandez, and A. Chahid, Phys. Rev. B 46, 6173 (1992), and references therein.

${ }^{10}$ Tables Annuelles de Constantes et Données Numériques, edited by Ch. Marie et al. (Gauthier-Villars, Paris, 1912), Vol. 3, p. 10 .
${ }^{11}$ S.R. Elliot, J. Phys. Condens. Matter 4, 7661 (1992); F.J. Bermejo, M. Garcia-Hernandez, F.J. Mompean, D. MacMorrow, and J.L. Martinez, Phys. Rev. B 51, 11932 (1995).

${ }^{12}$ F.J. Bermejo, A. Criado, A. de Andrés, E. Enciso, and H. Schober, Phys. Rev. B 53, 5259 (1996).

${ }^{13}$ M.A. Ramos and S. Vieira, in Nanostructured and NonCrystalline Materials, edited by M. Vazquez et al. (World Scientific, Singapore, 1995), p. 212. Also, M.A. Ramos, Q.W. Zhow, J.A. Moreno, and S. Vieira, in Proceedings of the Workshop on Non-equilibrium Phenomena in Supercooled Fluids, Glasses and Amorphous Materials, Pisa, Italy, 1995 (World Scientific, Singapore, in press).

${ }^{14}$ G.P. Johari, Philos. Mag. B 41, 41 (1980); G.P. Johari and J. Perez, Mol. Phys. 83, 235 (1994). 